\title{
ADDENDUM À \\ QUELQUES REMARQUES SUR DES QUESTIONS D'APPROXIMATION DIOPHANTIENNE
}

Patrice Philippon

Dans [1, Section 3], on a montré comment les conjectures les plus définitives sur les formes linéaires de logarithmes entraînent simplement la conjecture abc (dans sa forme faible). De façon plus pragmatique on peut également chercher les conjectures les plus accessibles sur les formes linéaires de logarithmes qui entraînent encore la conjecture $a b c$. De ce point de vue l'énoncé suivant semble être l'amélioration la plus minime de l'inégalité de Liouville qu'il conviendrait d'établir. On y note $|\cdot|_{p}$ la valeur absolue $p$-adique sur $Q$, normalisée de la façon habituelle ( $c$ 'est-à-dire, $|p|_{p}=1 / p$ ), et $h(\cdot)$ la hauteur logarithmique sur $\boldsymbol{Q}$ (c'est-à-dire, le logarithme du maximum des valeurs absolues des numérateur et dénominateur dans une écriture en fraction réduite).

Conjecture. Il existe des réels $0<\varepsilon<1 / 2, \alpha \geq 1, \beta \geq 0$ et un entier $B \in N^{*}$ tels que pour tous $x, y \in Q^{*}$ on ait $x y^{B}=-1$ ou :

$$
-\sum_{p \in S} \log \left|x y^{B}+1\right|_{p} \leq B \cdot\left(\alpha . h(x)+\varepsilon \cdot h(y)+(\alpha B+\varepsilon) \cdot \sum_{p \in S} \log p+(\alpha B+\varepsilon) \beta\right)
$$

où $S$ désigne l'ensemble des nombres premiers satisfaisant $\left|x y^{B}+1\right|_{p}<1$.

Nous déduisons de cette conjecture la version suivante de la conjecture $a b c$, où on reprend les paramètres $\varepsilon, \alpha, \beta$ et $B$ dont l'existence est conjecturée dans l'énoncé précédent.

ConsÉquence. Si $a, b, c \in N^{*}$ sont des entiers premiers entre eux satisfaisant $a+b=c$, alors $\log c \leq(B(\alpha B+\varepsilon) /(1-2 \varepsilon)) \cdot\left(\sum_{p \mid a b c} \log p+\beta\right)$.

Demonstration: On écrit $a / b=x y^{B}$ avec $x:=\prod_{p \mid a b} p^{v_{p}(a / b)-B\left[v_{p}(a / b) / B\right]}$ et $y:=$ $\prod_{p \mid a b} p^{\left[v_{p}(a / b) / B\right]}$, en désignant par $v_{p}$ la valuation $p$-adique et $[\cdot]$ la partie entière. On

Received 6th July, 1999

Copyright Clearance Centre, Inc. Serial-fee code: 0004-9727/00 \$A2.00+0.00. 
a $0 \neq x y^{B}+1=c / b, S=\left\{p ;\left|x y^{B}+1\right|_{p}<1\right\}=\{p \mid c\}$, la formule du produit et la conjecture ci-dessus impliquent :

$$
\begin{aligned}
\log c & =-\sum_{p \in S} \log \left|x y^{B}+1\right|_{p} \\
& \leq B\left(\alpha \cdot h(x)+\varepsilon \cdot h(y)+(\alpha B+\varepsilon) \cdot \sum_{v \in S} \log p+(\alpha B+\varepsilon) \beta\right) \\
& \leq B\left((\alpha B+\varepsilon) \cdot \sum_{p \mid a b c} \log p+\frac{\varepsilon}{B} \cdot \log (a b)+(\alpha B+\varepsilon) \beta\right)
\end{aligned}
$$

$\operatorname{car} h(x) \leq B \cdot \sum_{p \mid a b} \log p$ et $h(y) \leq B^{-1} \cdot \log (a b)+\sum_{p \mid a b} \log p$. Mais $a b \leq c^{2}$ d'où finalement

$$
\log c \leq B(\alpha B+\varepsilon) \cdot\left(\sum_{\boldsymbol{p} \mid a b c} \log p+\beta\right)+2 \varepsilon \cdot \log c
$$

et $\log c \leq(B(\alpha B+\varepsilon) /(1-2 \varepsilon)) \cdot\left(\sum_{p \mid \alpha b c} \log p+\beta\right)$.

REMARques. (1) Si on admet la conjecture ci-dessus avec seulement $0<\varepsilon<1$ et on suppose $a \leq b$, le raisonnement précédent montre

$$
\log c \leq \frac{\varepsilon}{1-\varepsilon} \cdot \log a+\frac{B(\alpha B+\varepsilon)}{1-\varepsilon} \cdot\left(\sum_{p \mid a b c} \log p+\beta\right),
$$

soit $* 2 / 3$ de $a b c$ * (forme faible). Par ailleurs l'énoncé de la conjecture pour $\varepsilon=1$ est une conséquence triviale de l'inégalité de Liouville.

(2) Dans la même veine on vérifiera (en décomposant $b / c=x y^{\mathcal{B}}$ plutôt que $a / b$ comme dans la démonstration ci-dessus) que la conjecture suivante :

Il existe des réels $0<\varepsilon<1 / 2, \alpha \geq 1, \beta \geq 0$ et $B \in N^{*}$ tels que pour tous $x, y \in Q^{*}$ satisfaisant $x y^{B} \neq 1$ on ait

$$
\log \left|x y^{B}-1\right| \geq-B(\alpha h(x)+\varepsilon h(y)+(\alpha B+\varepsilon) \beta) ;
$$

entraîne que pour tous entiers $0<a \leq b \leq c$ premiers entre eux et satisfaisant $a+b=c$ on a

$$
\log c \leq \frac{1}{1-2 \varepsilon} \cdot \log a+\frac{B(\alpha B+\varepsilon)}{1-2 \varepsilon} \cdot\left(\sum_{p \mid b c} \log p+\beta\right) .
$$


(3) Enfin, on vérifie facilement (voir [1, Section 3.b]) que la conjecture $a b c$, sous la forme : $\log c \leq K \cdot\left(\sum_{p \mid a b c} \log p+\gamma\right)$ avec $K>1$ et $\gamma \geq 0$ des réels, entraîne l'énoncé suivant pour tout $B \in N^{*}$ et tous $x, y \in Q^{*}$ tels que $x y^{B} \neq-1$ :

$$
-\sum_{p} \min \left(0 ; \log \left|x y^{B}+1\right|_{p}\right) \leq K \cdot\left(h(x)+h(y)+\sum_{p ;\left|x y^{B}+1\right|_{p}<1} \log p+\gamma\right) .
$$

En revanche, il ne semble pas clair que la conjecture $a b c$ (même dans sa forme forte) entraîne la conjecture de la remarque (2) précédente.

\section{RÉFÉRENCE}

[1] P. Philippon, 'Quelques remarques sur des questions d'approximation diophantienne', Bull. Austral. Math. Soc. 59 (1999), 323-334.

UMR 7586 du CNRS - Géométrie et Dynamique Université P. \& M. Curie

T.46-56, 5ème ét., F-75252 PARIS cedex 05

France 